\title{
Academic Experiences of "Zoom-Fatigue" as a Virtual Streaming Phenomenon During the COVID-19 Pandemic
}

\author{
Samual Amponsah, University of Ghana, Ghana \\ (iD) https://orcid.org/0000-0002-4303-4863 \\ Micheal M. van Wyk, University of South Africa, South Africa \\ (iD https://orcid.org/0000-0001-5536-1362
}

Michael Kojo Kolugu, University of Ghana, Ghana

\begin{abstract}
This phenomenological exploratory multiple-case study design was conducted at an open distance e-learning university and a traditional contact residential university, and it was found that the participants viewed video conferencing under the COVID-19 lockdown period as an exhausting experience. A second major finding revealed that the participants were empowered with digital literacy skills to use video conferencing effectively. The current findings add to a growing body of literature on video conferencing with a focus on Zoom fatigue. Further research might explore the lived Zoom experiences of administrators, students, and a larger group of faculties over a longer period. The study findings must be considered when planning and implementing video conferencing for academics and students in open distance e-learning contexts. This study showed that video conferencing is one tool in the emergence of a digital zoom revolution that has radically changed the workspace. The evidence from this study suggests that Zoom fatigue is a reality check for work-related health management.
\end{abstract}

\section{KEYWORDS}

COVID-19 Pandemic, Multiple Case Study Design, Open Distance E-Learning, Phenomenology, Video Conferencing, Zoom Fatigue

\section{INTRODUCTION}

Since late 2019 the COVID-19 pandemic has changed the face of human interactions in everyday life. Corbera, et al. (2020) highlighted that we are now living through a global pandemic with unprecedented scope, scale and impact and no industry or sector remain unscathed. The authors also highlighted that the speed at which the pandemic is travelling across boundaries is unparalleled; therefore, they anticipate dire consequences for all aspects of life in the near and unforeseen future. Additionally, it is worth noting that beyond the pandemic, things are not likely to return to their former state. Consequently, the world needs to accept the new normal and find ways to survive during and postCOVID-19. The Commonwealth of Learning (COL) (2020) thus considers it imperative to venture into uncharted terrain to survive and manage the probable impacts of the pandemic.

The education sector which transcends borders, status, colour or creed has been hit hard by the virus. Based on the statistics provided by COL (2020), it is estimated that over 90\% of the world's

\section{DOI: 10.4018/IJWLTT.287555}

This article published as an Open Access article distributed under the terms of the Creative Commons Attribution License (http://creativecommons.org/licenses/by/4.0/) which permits unrestricted use, distribution, and production in any medium, provided the author of the original work and original publication source are properly credited. 
student population has been sent home and has stayed out of school since the pandemic became a global crisis. To confirm the position of the COL, the United Nations (2020) puts the figures of children and youth who are out of school due to the COVID-19 pandemic at 1.6 million globally. Despite the shutdowns, the educational sector needs to thrive as it serves as the bedrock on which other sectors may survive. Corbera, et al (2020) thus advocate for the need to learn new ways of operating. Additionally, the COL (2020) has also documented that the separation in space and time between faculty members and students' calls for using some form of technology or media that will restore communication between the two parties.

The need to leverage on technology to mediate communication between faculty and students and among faculties has seen a spike in the use of video conferencing applications, especially during the COVID-19 pandemic (Tanis, 2020). One video conferencing application that continues to see significant usage in this time is Zoom. According to the World Economic Forum (2020), the Zoom video conference facility was downloaded 2.1, 4.3 and 27 million times respectively in January, February and March 2020. Though the organisation has indicated that the application is not the most used, they christened it the rising star among video chat applications due to the increasing number of people logging on to it daily. Although the World Economic Forum (2020) has not touted Zoom as the most used videoconferencing facility, we are interested in its usage owing to its high adoption rate by faculties in recent times and want to gauge their experiences with its usage during the COVID-19 pandemic. Our interest finds roots in Reisinger's (2020) finding that Zoom had nearly two million users in January and February 2020.

Like many other internet-based infrastructures, Zoom has immense benefits, especially during the time of social distancing and lockdowns in many places in the world. As noted earlier, it has the propensity of bridging the communication gap between faculty and students and among faculty members. We believe that it will continue to sustain communication even post-COVID-19.

However, this does not make it immune to the challenges that users might encounter; or may already be encountering when zooming (thus, using the Zoom video application for videoconferencing). Hence, this reflective paper reports on academics' experiences of an online phenomenon, "zoom fatigue" when attending online video conferencing meetings. This paper conceptualises the term "academic" in line with what is offered by the Marriam-Webster Dictionary (n.d.) as a member of a university or college who teaches and conducts research. As authors of this paper, we extended this definition that an academic is appointed in an academic department and is responsible for key performance areas such as tuition, research, community engagement, academic citizenship and if promoted to a senior academic position, including academic leadership.

This paper aims to explore academics' views of an online behavioural-related issue-zoom fatigueand provide remedial strategies to overcome zoom fatigue in video conferencing. The purpose is based on what we have gleaned from the literature that zooming comes with various stressors which have come to be known as zoom fatigue. And also on the back of some faculty members using unfamiliar tools and unpractised skills which may increase their cognitive load and fatigue. Agreeably, both sides of the usage of the application have been well-documented albeit not in the Ghanaian context and not much in the South African context either. As our contribution to the knowledge, practice and theory of adopting and using the Zoom video conferencing application, we sought the views of faculties at higher education institutions in South Africa and Ghana.

The following questions were specifically formulated to explore the main aim of this paper:

- Why is the COVID-19 lockdown online video-conferencing a behavioural phenomenon?

- What are academics' experiences related to zoom-fatigue in online video-conferencing?

- What strategies can be employed to remedy zoom-fatigue in online virtual streaming conferencing? 


\section{LITERATURE REVIEW}

\section{Zoom Fatigue}

There is no doubt that Zoom has saved many educational institutions from being locked down on communication due to the lockdowns and social distancing protocols enforced in recent times. However, a study conducted on the emotional state of persons has established that users experience mental exhaustion with sustained zooming (van der Linden and Eling, 2006). Moreover, Hines and Sun (2020) refer to this experience as zoom exhaustion. The scholars further explained that, "Unnatural, unexpected and annoying sounds invoke a response in our brains and force us to concentrate on them" (p. 2). In essence, they indicated that the slightest sound besides that which is central to a virtual meeting is likely to contribute to people's zoom fatigue. Contrariwise, silence during zooming also contributes to zoom fatigue. In this light, Jiang (2020) is quoted as saying, "Silence creates a natural rhythm in a real-life conversation. However, when it happens in a video call, you become anxious about technology” (p.2).

The amount of time spent on zooming is regarded as a factor contributing to zoom fatigue. For instance, Spicer (2020, p. 2) argued as follows: 'But all this time spent on video calls has its problems.' He indicates that all the time spent on zooming leave users feeling tired and empty as the physical interactions remain absent. He, therefore, indicates that zooming has spurred on a new form of psychological affliction called zoom fatigue. In corroborating this form of fatigue, Jiang's (2020) engagement with a professor and an expert on the matter showed that zooming required more focus than face-to-face interaction. The explanation is that one has to do more to understand the non-verbal gestures and mimic a real-life conversation (Jiang, 2020; Spicer, 2020).

Further to this argument, it has been revealed that screen time deserves some attention. Although in a publication by the International Baccalaureate Organization (IBO) (2020), it has been asserted that using videos in conferencing does not count towards screen time (the amount of time spent on devices to access onscreen activities). The document also claims that from age six there is no limit to the duration of screen time. We, however, find these inimical to sustained zooming and a key contributor to zoom fatigue. Our argument is skewed towards the issues identified in the preceding paragraph and on the basis that Spicer (2020) and AL Ma'mari, et al. (2020) recognised that emotional exhaustion, fatigue and burnout occur because of overload and exposure to zooming. Thus, the energy one needs to concentrate on grasping all that is happening in the virtual space.

Another angle that is introduced into the debate is that zooming can be emotionally exhausting. To this, Spicer (2020) described how a group of therapists who conducted sessions through video conferencing indicated that they had 'lost connections' with their clients. This shows how a lack of physical presence can affect people and the fact that zooming and other videoconferencing facilities cannot fill the physical vacuum created. Spicer also argued that students may feel anxious as they feel they are filling a big stage while workers may feel exiled and may do extra to attract the attention of managers in virtual space. We add that the pressure is not only on students and those of lower rank but academics and managers would also have to go that extra mile to ensure the success of events held on Zoom. Moreover, Shuttleworth (2020) reported in a case study that too much work and sleeplessness could increase emotional exhaustion and fatigue. In this regard, Zoom fatigue affects superiors and subordinates in their bid to do their best while zooming. The IBO document (2020) aptly captures that there is pressure on people to overperform while zooming.

One of the experts interviewed by Jiang (2020) hinted that being in large group chats on Zoom can be 'depersonalising'. He explains that such large groups chatting in virtual space diminishes one's power as an individual. This is agreeable as having too many eyes focused on you could bring pressure on you, except when you are very experienced in what you do and are familiar with zooming. This kind of pressure is similar to what Spicer (2020) referred to as the feeling of being overlooked by others. It gets even worse when the eyes of your superiors are fixed on you. This situation connects to the emotional exhaustion of performing beyond the ordinary. 
Lastly, technical issues also contribute to zoom fatigue. On that, Jiang (2020) mentioned that the freezing of screens of the gadgets used when zooming tends to contribute to zoom fatigue. Users' perceptions of insecurity when controlling data and recordings, among others, are also contributory factors to people's zoom-fatigue (Lawson, 2020; Reisinger, 2020). Jiang (2020) codes this experience as Zoom bombing or hijacking as it mostly happens unexpectedly. Additionally, the frustration some people, especially students and lower-earning workers, have to experience to get [enough] data for zooming in itself causes fatigue (van der Linden and Eling, 2006). Owing to data issues, such as the high cost of data, Tlou (2020) reported that private university students in South Africa are unable to study. Apart from data unavailability, the IBO (2020) document described how poor bandwidth and connectivity can be stressful during synchronous sessions.

\section{Remedying Zoom Fatigue}

The preceding section explored factors contributing to zoom fatigue. It is common knowledge that problem identification and understanding form the initial stages of problem-solving. As a result, this section presents some practical solutions that have been gleaned from available literature to help remedy the situation. Firstly, it has been noted that it is always advisable to mute microphones not to irritate others while zooming except when one wants to contribute to the session (Hines and Sun, 2020; Jiang, 2020). Besides muting the microphone and videos, Hines and Sun (2020) have added the extra caution of not mumbling behind our gadgets. They also touched on arranging spaces in such a way that disruptions would be minimised.

Scholars shared that we should cut our cognitive workload and ensure maximum concentration by avoiding multitasking while zooming (Spicer, 2020; Cross and Polk, 2018). This may seem impossible to do as faculty members and students are adults who mostly multitask (OFCOM, 2014). He, however, added that taking intermittent breaks can ease zoom fatigue. Moreover, Halupa and Bollinger (2020) reported that academics mitigated levels of fatigue using technology-free zones and off-screen timebreaks. On the part of those who schedule Zoom meetings, the IBO (2020) document and van der Linden and Eling (2006) recommended that the duration of activities be limited with clear goals to be achieved. We believe that heeding to the recommendation of the IBO would condition the minds of participants to prepare and participate while limiting or eliminating zoom fatigue.

As indicated in the introductory section of this paper, zooming is in vogue, especially during the COVID-19 pandemic, but its downsides have also come into play. It is in this direction that researchers have indicated that a clear path of sidestepping the situation is finding alternative ways of communicating. Langford and Damsa (2020) agreed with Spicer (2020) that text messaging, emails and phone calls are believed to be effective alternatives to zooming. They even believe that using handwritten thank you notes has therapeutic benefits for the recipients. Jiang (2020) and Pettersson (2018) add that using the chatting tool on zoom helps to reduce zoom fatigue. On this note, one of the experts interviewed by Jiang indicated that sharing files with clear notes [ahead of zoom] meetings could also help to avoid information overload.

Because of factors, such as depersonalisation and emotional exhaustion that participants may be battling with while zooming, experts have advocated that video cameras on gadgets could be turned off. Jiang (2020) feels this can aid concentration while Spicer (2020, p. 3) writes, 'Hiding the image of yourself during a video conference can make you feel less self-conscious and more focused on what others are saying'. Jiang (2020) believes that we do not always have to be formal while zooming. Both authors, thus, suggest that participants can spend some time checking on one another's wellbeing and catching up before moving onto actual business. He adds, 'It's a way to reconnect us with the world and to maintain trust and reduce fatigue and concerns' (p. 5). In this light, Vetter (2018) argued for the use of convivial technologies which Macgilchrist (2020) explained as technologies that go handin-hand with changes that occur in social relationships. From the researchers' point of view, UNISA stands out as deploying convivial technologies better than the University of Ghana (UG); so, this solution might not work in both contexts at the same level and speed. 
Lastly, on the technical issues that contribute to zoom fatigue, Reisinger's (2020) research has established that zoom security has improved. Nonetheless, he advocates that users should integrate security features and ensure their virtual protection. The reduced data rate arrangements made by South African public universities (Tlou, 2020) could be a good model to leverage for private schools and other parties for the sake of those who cannot afford data. In the instance of Ghana, the two leading telcos (MTN and Vodafone) zero-rated activities that were routed through the Sakai Learning Management System (University of Ghana, 2020). However, this did not include the Zoom video facility. Which means faculty members and students could save the data that would have been expended on the Sakai Learning Management System for zooming. This falls in line with Hines and Sun's (2020) advice that while videoconferencing we should ensure that the bandwidth is not consumed by other users or facilities.

Based on the explication of the literature on what zooming fatigue represents and how it be remedied, an important theory that can underpin this study is the Job-Demand Resource Theory (JDR Theory). In this theory, Lazarus and Folkman (1984) define stress as a situation where external factors disrupt the equilibrium of cognitive, emotional and environmental systems as witnessed in the COVID-19 period. Because life goes on even in dire situations as we find ourselves under the COVID-19 pandemic, Demernouti et al. (2001) make a connection between such disruptions and job demands by indicating that 'job demands refer to those physical, social, or organisational aspects of the job that require sustained or mental effort and are therefore associated with certain physiological and psychological costs (p. 501). This resonates in the contexts of this study as the faculty members in this study had to brace themselves against all odds to complete their semester's work. The completion of their work did not happen in a vacuum. Ritcher and Hacker (1984) emphasised the need for resources if one can lessen stress and meet job demands, especially, in such emergency times. They, therefore, identified internal resources as important in succeeding. This, they explained as the individual's cognitive features and capabilities as well as action patterns that position them for success or otherwise. The other form of resource, external, is described by Demerouti et al. (2001) as the physical provision and the social support provided by the institution as support for employees. Hockey (1993) associates the resources to the health of the employee and averred that when these resources are made available, employees would not be overtaxed and would be motivated to work.

\section{Context Of The Multiple-Case Study}

As indicated earlier, academics are responsible for key performance areas (job description). Each job description according to the level of appointment (from junior lecturer to tenured professor) must be executed and measured over time. In this multiple-case study, academics perform tasks related to teaching, learning, assessment, student support, research, community engagement, outreach, academic citizenship as well as mentoring and coaching. In the context of this paper, these performance areas are executed in a different context (open distance education and face-to-face/contact residential).

The University of South Africa (UNISA) is an African open distance e-learning (ODeL) university with the largest student population on the continent. It is the front runner in open distance education in Africa with several university partnerships and similar global distance education universities. This ODeL university offers multiple online courses, degrees and certificates through a blended to an online learning platform that is accessible and open to all students, locally and internationally. Academics, admin staff and students are connected for online engagements at any time, everywhere and any place as a means of advancing online learning. The academic staff and students at the ODeL university access the learning management system (LMS) for all academic and non-academic activities. But the COVID-19 lockdown changed the manner in which this ODeL University conducts meetings. Suddenly they were forced to use Microsoft Teams for departmental meetings, faculty committee meetings and faculty board meetings.

On the other hand, the University of Ghana (UG) was established in 1948 and has a rich tradition with alumni which is globally recognised. UG is the premier and largest university in Ghana in terms 
of size and student population. Its student population stands at 38000 including international students from over 70 countries (University of Ghana, 2020). In 2014 the University deployed the Sakai Learning Management System as a way of moving away from the traditional forms of delivery to a hybrid system (Amponsah, Badu-Nyarko, Obodai and Anane, 2019). However, while the Distance Education Department leveraged on the LMS, the mainstream largely continued with the traditional means of delivery. Incidentally, the advent of the COVID-19 pandemic-motivated lockdowns and social distancing protocols impelled the University Management to enforce training of all faculties in using the LMS to continuously engage students. Consequently, this witnessed an upsurge in the use of the Zoom videoconferencing facility.

\section{Methodology}

This paper employed a phenomenological research paradigm as a lens to capture the lived experiences of eight academics who participated in several video conferences for departmental meetings, committee meetings, webinars, online professional development sessions, online teaching and online alternatives assessment during the COVID-19 lockdown period. The basis for the use of this design hinges on its strength in exploring and discussing phenomena from the perspective of those who 'live' it (van Wyk and Taole, 2015). Though all the researchers in this study are academics who have been involved in videoconferencing, we were guided by the principle of the phenomenological design to bracket our experiences so we could fully present the perspectives of the study subjects. This exploratory phenomenological multiple-case study design was conducted at two African universities. One university is an open distance e-learning university (ODeL) located in South Africa and the other is a traditional contact residential university located in Ghana. Before conducting the research, ethical clearance was applied and granted by the university's ethics committee (Reference 2020/05/13/90178912/18/AM). The university policy stipulated that confidentiality and adherence to COVID-19 protocol be observed and adhered to before, during and after the COVID-19 lockdown. When the COVID-19 pandemic occasioned a shift to online learning, we sent emails and texts to faculty members in our universities to invite them to participate in this study but after a month we only received a handful of positive responses. We assumed the pressure of shifting online caused the non-responsiveness so we followed up with in-person and telephone calls which yielded 12 and seven responses from UG and UNISA respectively. Initial interactions with the 19 who accepted to participate in the study showed that seven of them were occasional video conference users and were attendees on the occasions they participated so could not help in fully responding to the field questions. Four out of the remaining participants also proved to be too difficult to reach as their schedules pitched them against their willingness to participate. After several weeks of their inability to participate, we were left with only four academics from each university to participate in this study. The participants from UNISA comprised two males and two females while UG had three males and one female. The selection of the eight was based on their readiness and availability and also being in a video conference at least twice a week. Besides, they all agreed to have organised a video conference and chaired at least one video conferencing meeting. Their experiences were important in answering the research questions guiding this study. Online interviews, telephonic conversations and Microsoft Team meetings were selected as tools for data collection. The data collected was downloaded from the online interviews, telephonic conversations and Microsoft Team recordings. All these data sets were transcribed and themes were identified. The data transcriptions were done individually by each researcher after which they compared notes to ensure the transcriptions were accurate. The same process was followed in arriving at the themes that emerged from the analysed field data. The data analysis and transcription process was done during the COVID lockdown period.

\section{Results}

- Why is the COVID-19 lockdown online video-conferencing a behavioural phenomenon? 


\section{"Zoom Fatigue" as a Physical, Emotional and Energy- Draining COVID-19 Phenomenon}

The majority of participants (90\%) agreed that "zoom fatigue" is an 'emotional and energy-draining action', a state of impacting on the body and the mind. They viewed concerns that some meetings organised during COVID-19 were unplanned by line managers and committee chairs. This type of work arrangement was a new type of communication in an online space during the lockdown. Many viewed attending video conferences for longer than an hour; watching and listening to speakers stating points on issues as an 'online-taxing exercise'. Some also said it is physically and emotionally exhausting during video conferencing. One participant said the following in this regard: 'Yah, I am so physically drained, I felt the fatigue - [sic] I felt so tired and my head was spinning after the long sessions during our meetings and workshops'. Some of these views were extracted from the data sets. One participant stated the following: 'I am feeling so tired, burned-out, and exhausted'. The participants voiced their frustrations: 'If you are tired you cannot listen anymore. You lose your focus on issues during the discussion'. One said: 'Yah, I am so fatigued - [sic] I felt so tired and my head is spinning'.

\section{Online Video Conferencing as Attentiveness and an Attraction With Direct Visual Contact}

The participants were asked how they experienced video conferencing. They felt that the COVID-19 lockdown period forced them to use online videoconferencing platforms as a way to meet. One participant said: 'It is attentiveness and visual-contacts phenomenon that came and will stay with us. It had to change the way we meet and communicate'. One participant responded in this regard: 'The Teams meeting is a video-conference that you need to focus on, get your attention. Your eyes are fixed to a flat-screen. It can be straining. Ultimately, it is you that must keep track of visuals and your concentration levels'. Many mentioned that video conferencing is straining on the eyes but eye contact always follows the visual and voice tone of the presenter. Some said: 'It was very hard to pay attention to some presenters during the online conference sessions'.

- What are academics' experiences related to "zoom-fatigue" in a virtual streaming videoconferencing?

\section{Empowered with Digital Literacy Skills and Knowledge of Online Teaching}

Many participants said they had prior digital literacy skills and knowledge of online teaching. They said Teams is a video conferencing strategy which is a spontaneous online teaching experience. One participant said the following in this regard: My experience with Zoom or Teams as an online platform to hold meetings or for teaching has sharpened my knowledge base with such technologies. Similarly, another participant mentioned that 'Teams had a positive impact on my knowledge of how to use these and other online tools. Now that we use Teams for meetings and teaching, it is an involuntary teaching platform'.

\section{Experiencing an Accelerated Zoom Revolution Movement}

One participant shared how the COVID-19 lockdown had digitalised online learning and impacted on how the will meet, teach and socialise in the year ahead and beyond. He posted the following on Teams: 'Yes, my view is that since this COVID-19 lockdown started, we experienced an accelerated period of online meetings, teaching, and socialization, exponentially'. Another academic wrote: 'The influence of what I cal zooming expansion will move together within the 4IR space. We will see a much higher level of increase in using Teams, Zoom meetings, WhatsApp, and other online platforms for meetings. Post-COVID-19 lockdown will never be the same - it will be business unusual'. 


\section{Empowered with Digital Literacy Skills Using Video- Conferencing (Teams or Zoom Meetings)}

A male participant shared a response in appreciation of the new digital skills learned stating the following: 'This Microsoft Teams as a video-conferencing tool was introduced to us at a university continuous professional development session. I also attended two follow-up sessions this year. I am delighted to be reskilled digitally. I started to experiment with it last year and now I am proudly using it with my students during COVID-19 lockdown'. A female participant shared her joy as she narrated how she overcame her initial stress to be able to use the video conferencing facilities. She said, 'I was stressed out mostly but after attending the university-organised training, I confidently organise zoom meetings and occasionally chair some of the meetings. I have been coaching some faculty members on videoconferencing lately.'

\section{Personal Lived Experienced Created Excitement and Appreciation}

Following the interview sessions conducted online or telephonically, the participants expressed their appreciation. Those academics who attended the COVID-19 Teams meetings through videoconferencing expressed excitement and appreciation. A participant reflected: 'I appreciate that we could hold some meetings on Teams. This lockdown is very frustrating, but I appreciate that we could discuss important academic issues. I am excited that at least we met on this platform to discuss what we will do after lockdown'. Another participant shared her challenges with video conferencing but was appreciative of it at the same time. She shared the following, 'I can't deny the data issues and technical challenges yet I wonder how schools would have survived without these platforms. I have successfully completed the semester and all other engagements through the Zoom platform.'

\section{Enhancing Cognitive Workload and Span of Concentration Level During Online Meetings}

One of the online video conferencing interviewees said the following: 'Sometimes we are overloaded with too much cognitive workload, but we have gotten used to it now'. That is a positive instead of a negative. Another ODeL academic said: "yes, working at a distance is common for me but COVID protocols and regulations had impacted my work emotionally... yah is very stressful during these times" Highlighting what is good about videoconferencing, one of the female participants said: 'You need to think and listen at the same time. I am used to multitasking'. Another participant said: 'Most of our meetings are forcing us to ensure maximum concentration. This is a very good and new skill for me. You need to concentrate otherwise you lose track of the presentation'.

In spite of the positive remarks of video conferencing, there were also some disappointments among participants regarding this phenomenon as reflected in what follows.

\section{Inconsistent Connectivity and Digital Challenges}

Participants alluded to poor sound quality, break-ups in connectivity and the low quality of video conferencing which has an impact on ears and hearing. This participant said: 'At my first Team meeting, I was disturbed about how people behaved. I recall how the chair of our meeting kept reminding academics to mute their microphones due to disturbances from those who had not muted their microphones'. These words always echoed in my ears, said a female participant: 'I reminded colleagues to mute their mics, switch-off their cameras. I could not hear you, you are breaking up. What are you saying, just repeat what you are saying'. In most of the interview sessions, these sentiments were posted or expressed. Most participants posted their disappointment in 'poor presentations, poor sound, and high noise levels were disturbing and break their concentration'. One of the participants made the following statement: 'I hate to sit in meetings whereby poor quality presentations or background noise influence my concentration'. 


\section{Videoconferencing is a Time-Wasting Exercise and a Costly Activity}

One male academic participant posted his response during the telephonic interview: 'It felt that COVID-19 lockdown had to change our meetings to online chatrooms - some people discuss items that were not on the agenda - this is time-wasting '. Another participant expressed his disappointment: 'I think online conferencing is time-wasting when I have to sit and listen for hours to presentations. I could be more productive. This is exhausting'.

Given the perspectives shared thus far, there was the need to tap into the minds of the participants on how best they thought zoom fatigue could be minimised or eradicated. Their responses are captured under Research Question 3 below.

- What strategies can be employed to remedy zoom-fatigue in online virtual streaming conferencing?

\section{Reducing cognitive load through efficient shorter online video conferences}

When asked for their opinions on how zoom-fatigue could be remedied, the participants touched on several strategies. First, they felt that if meetings are kept shorter [maximum of an hour] and they are not held too often, it would relieve them from mental exhaustion and drain on their energies. One participant noted the following: 'Having shorter meetings on zoom will be helpful'. In terms of the frequency of meetings, most participants agreed that Zoom meetings should not be held too frequently. In this vein, one participant said: 'I think having fewer zoom meetings in a week will be fine'. A participant summed up the views collated in this statement: 'Only schedule or attend important meetings'. To help keep to the duration of scheduled Zoom meetings, one participant suggested the following: 'Meeting etiquettes of moderators exhausting agendum and arriving at resolutions should be adhered to in order to achieve zoom meeting goals'. To achieve results from Zoom meetings, a participant proposed: 'We could even have follow-up meetings to track our decisions of all our meetings'.

\section{Remedying Through Effective Moderation of Meetings}

The participants were of the view that the chair or moderator of Zoom meetings could do a lot to reduce zoom fatigue among participants during meetings. It comes with little surprise that all participants indicated that the chair should introduce breaks. For instance, a participant shared: 'Chairs should introduce breaks in between sessions to help people cool off and engage themselves outside the issues tabled for the meeting'. Another participant further underscored the importance of breaks by saying: 'Chairs of meetings should take frequent breaks for attendants' mental relaxation'. Besides breaks, one participant was of the view that: 'Discussions should be moderated in zoom meetings since some members seem to deviate from agenda/subjects along the way'. Another strategy is to plan breakaway periods or setting time-out from online video conferencing meetings. Furthermore, plan for a couple of days without Zoom meetings to recover from back-to-back video conferencing meetings.

\section{Eliminating Disruptions Through Self and Collegial Support}

All the participants admitted that it was possible to eliminate the noise that normally disrupts video conference meetings and add to the fatigue of those in the meetings. Half the participants agreed that they could control their microphones and video cameras not to distract others. Some also placed the responsibility on the meeting host. A participant reacted as follows: 'Control of microphones by the host should be managed very well'. Beyond the gadgets, a participant shared the following: 'I suggest a reorientation of minds for participants to get accustomed to this way of life'. The idea is that when individuals are reoriented towards the new paradigm, they would act ethically. In a similar stance, one of the participants called for collegial support by stating as follows: 'The institutions that opt for the video-conferencing mode of interactions should take their participants through ways to 
effectively use the tool to avoid the distractions and interruptions from some members'. Essentially, the participants would act ethically while participating in video conferences.

\section{Discussion of Findings}

This exploratory study attempted to answer three research questions related to academics attending online videoconferencing as a zoom fatigue phenomenon. To develop this inquiry, we used a phenomenological lens to capture the lived experiences of academics who participated in this study. Thus, academics' experiences of the online phenomenon, 'zoom fatigue' was investigated and ultimately strategies were provided to overcome zoom-fatigue in online video conferencing.

The first question focussed on academics' views regarding why online video conferencing is a physical and emotional phenomenon. Academics expressed 'zoom fatigue' as an 'emotional and energy-draining action', which impacts on the body and soul. Given the latter, van der Linden and Eling (2006) reported that local processing was affected by fatigue that impacts on the attentiveness of the person to complete the task or assignment at hand. Furthermore, according to them, it is a physical and emotional experience that is "physically draining and exhausting because of long meetings and workshops'. In addition, Coetzee et al (2019) reported that chronic fatigue and burn-out are two major issues experienced by academics. This view is supported by Al Ma'mari et al. (2020) who state that fatigue had a significant effect on critical care nurses' safety in the workplace. Both multisites academics echoed lived experiences of a virtual fatigue presence. But the academics who were used to the norm to execute their responsibilities at the ODeL space only came under COVID-19 lockdown regulations which were different. To this one academic echoed... "Yes, working at a distance is common for me but COVID protocols and regulations have impacted my work emotionally... yah is very stressful during these times". Kubey et al. (2001) reported that Internet use and academic performance is influenced by the applications of virtual spaces. On a positive note, academics viewed Microsoft Teams as an online videoconferencing tool that require them to focus attentively whether attending meetings, having conversations or participating in online training sessions. For example, Chatwin and McEnvoy (2019) used online videoconferencing to train carers in the UK and reported that the participants were positive about the online tool preparing them as carers. The participants in this study felt that the COVID-19 lockdown period forced them to use online video conferencing platforms as a way to meet or train. This view is supported by several scholarly works that motivation and mood, specifically in an online space will impact on the attention of a participant (Abbass et al. 2011; Derryberry and Tucker, 1994; Hines and Sun, 2020; van der Linden and Eling, 2006). Situating the responses in here in the JD-R Theory shows that participants leveraged their internal resources (cognitive features and action patterns) to minimise their job demands and any stress the sudden shift might have caused them (Hockey, 1993; Lazarus and Folkman, 1984; Ritcher and Hacker, 1998). In sum, the COVID-19 lockdown protocols and regulations had forced both institutions to increase the use of virtual streaming (Teams or Zoom) for teaching and holding meetings. The COVID-19 regulations of wearing masks, social distancing, and health consciousness created a "new normal" which had a knock-on effect on teaching or attending meetings by academics. Both multi-site academics experienced this "new normal" as stressful and emotional draining experience.

The second question dealt with academics' experiences related to online video conferencing. As online facilitators, many participants said they had prior digital literacy skills and knowledge of online teaching. This again resonates the internal resources in the JD-R Theory as explained by Ritcher and Hacker (1998). A study conducted by Ip (2011), using video conferencing to teach intercultural communicative skills to foreign language students reported significant results for future studies. Besides, Sato et al. (2019) reported that the university project team developed online digital literacy resources for academics to use in online courses. In this study, several participants viewed digital literacy skills and online facilitation as important competencies required to be effective, specifically when using Teams as a video conferencing strategy. Several studies reported the educational advantages of using video conferencing and web-based learning as virtual learning spaces for training students 
and academics (Hobbs and Coiro, 2019; Jayaraman and Jothiswaran, 2020). For the participants, Teams is one of the many online video conferencing tools that created an impetus and accelerated Zoom Revolution Movement (ZRM). A participant alluded that 'zooming', as a movement in an online space, had revolutionised the knowledge economy exponentially. The ZRM had digitalised online learning. It impacts on how faculty members will meet, teach and socialise. This participant concluded that: 'Since this COVID-19 lockdown, we have experienced an accelerated period of online meetings, teaching, and socialisation, exponentially'. As stated earlier, participants alluded that they were empowered with digital literacy skills, using videoconferencing [Teams or Zoom meetings]. Some of them expressed an appreciation for new digital skills learned and posted that: 'Microsoft Teams as a video-conferencing tool was introduced to us at a university continuous professional development session'. Scholarly texts concur that institutions of higher learning must take advantage of online platforms to empower staff and students with digital literacy competencies (Hofflander et al. 2019). The provision of such platforms is supported by the JD-Theory element of external resources wherein Demerouti et al (2001) argue that the absence of such support leads to employee demotivation and eventual burnout.

During lockdown and emergency periods, it is of vital importance to create continuous professional development opportunities for academics (Hobbs and Coiro, 2016; 2019; Jayaraman and Jothiswaran, 2020). For the participants, collegial support in planning efficient shorter online videoconferences is important because of busy schedules in an online context. Some participants proposed that the Zoom meeting hosts and organisers plan shorter and more frequent meetings on the Teams platform. To this one participant's expression states that: 'I would prefer that we plan more frequent and shorter meetings'. Studies show that two critical factors impacting on planning and implementing video conferencing are the time dimension (Hofflander et al. 2019) and the human factor (Oeppen et al., 2020) in online training.

Despite the positive views expressed by the participants and a plethora of studies on videoconferencing, several drawbacks were highlighted. Inconsistent connectivity and digital challenges were identified in most of the responses. The participants alluded to poor sound quality, break-ups in connectivity and the low quality of video conferencing which has an impact on ears and hearing. A participant posted that video conferencing is a waste of time and a costly activity. The following opinion was posted: 'Our meetings to online chatrooms - some people discuss items that were not on the agenda - this is time-wasting '. In Conversation, an academic online conversation space, Hess and Sun (2020) published a brief conversational review on how to make video conferencing less tiring. Several scholars posit that the quality of streaming of video conferencing, using Microsoft Teams meetings or training sessions could affect the clarity of online audio-visuals if participants do not mute microphones and switch off video cameras (Hess and Sun, 2020; Jiang, 2020; Spicer, 2020; Oeppen et al. 2020).

The final research question sought to elicit strategies that can be employed to remedy zoom fatigue in online conferencing spaces. It was proposed that the cognitive load of participants in videoconferencing could be reduced through efficient shorter online video conferencing. The participants felt if meetings were kept shorter [maximum of an hour] and were not held often, it would relieve them from the mental exhaustion and drain of their energy. These findings are supported by Cross and Polk (2018) that provided useful advice on how to manage and overcome burn-out, stress and fatigue in the virtual streaming space. Furthermore, Halupa and Bollinger (2020) conducted a multiplesite design study at three universities amongst academics in online spaces. It was concluded that participants mitigated online fatigue strategies by using technology-free zones or virtual time-breaks. One participant noted the following: 'Having shorter training sessions will be helpful with cognitive overload'. This view is supported by Hess and Sun (2020) on how to make video conferencing less taxing and suggested scheduling shorter online meetings. Second, remedying through effective moderation of meetings for effective participation in the online space (Macgilchrist, 2020). The participants were of the view that the moderator of video conference meetings could do a lot to reduce 
zoom fatigue among participants with short breaks during meetings. Finally, eliminating disruptions through self-support and collegial support will facilitate communication on an online platform. All the participants admitted that it was possible to eliminate the noise that normally disrupts Zoom meetings. An organiser or participants would limit disruptions during meetings or training sessions by controlling microphones and video cameras (Hess and Sun, 2020; IBO, 2020 van; der Linden and Eling, 2006). The JD-R Theory is emphatic on how job demands could be stressful and lead to employee burnout in the absence of organisational social support. Hence, the necessary action from the organisers of video conferencing meetings and the participants (self and collegial support) are deemed to have far-reaching implications for remedying zoom fatigue.

From the analysis of the field data, it was adduced that though the multi-sites - in terms of their established mode of delivery - differ, the nature, medium and circumstances of the majority of the tasks of academics where somewhat similar prior to the outbreak of the COVID-19 pandemic. In that faculty meetings were done in a face-to-face manner; faculty worked from their institutional offices, and students had access to their institutional conducive academic facilities for interaction with their instructors. But the unexpected impact of COVID-19 forced these two institutions to heavily adopt virtual streaming means for all academic and administrative tasks. Thus, the effect of the exigencies of the complete movement to the virtual video-conferencing environment would be felt by academics from the two institutions howbeit slightly dissimilar.

\section{Conclusion}

In this investigation, the aim was to assess academics' lived experiences of an online behavioural phenomenon, zoom-fatigue, and suggest strategies to overcome video conference fatigue. This study has found that participants viewed video conferencing under the COVID-19 lockdown period as an exhausting experience that affects the quality of their physical and emotional dimensions of life. The multi-site academics agree that teaching or attending meetings using virtual streaming platforms under COVID-19 had a stressful and emotional taxing experience. A second major finding revealed that participants were empowered with digital literacy skills to use video conferencing effectively. Findings showed that 'zoom-fatigue' is viewed as a physical, emotional and energy-draining COVID-19 phenomenon. Also, the faculty agreed that video conferencing is one tool in the emergence of a digital zoom revolution that has changed the workspace exponentially. Nonetheless, several drawbacks were highlighted which need further inquiry. The evidence from this study suggests that zoom fatigue is a reality and the findings of this study must be studied in planning and implementing video conferencing for academics and students in a distance education context. Significantly, this study has explained how job demands in critical and emergency times, such as the COVID-19 pandemic, could lead to stress among faculty members. However, as emphasised in the JD-R Theory, resource availability is beneficial in reducing stress and eventual burnout while the converse situation holds. In the context of this study, internal resources (cognitive features and action patterns) of the faculty members served as their motivation which in turn helped to reduce their cognitive load in the face of the challenges. The current findings add to a growing body of literature on videoconferencing with a focus on zoom-fatigue whether in synchronous or asynchronous contexts. Also, the findings suggest that if the following recommendations are considered and included as features on video-conferencing applications they would help reduce video-conference fatigue: Automatic noise muting or alerting system; fatigue detection systems using either voice or facial cues or both; A feature that allows the scheduling of time allotment for individual agenda and breaks before the start of the meeting or during the scheduling of the meeting, this would serve to alert participants and enforce breaks; and also a feature that allows a presenter to hear himself or herself as other participants would, this would help the presenter deal with the silence in video-conference meeting where all the mics are muted except that of the presenter. Also, institutions should make efforts to train their faculty on the appropriate use of web-based video conferencing facilities while making policies to address the challenges they face in using such applications. The scope of this study was limited in terms of the 
number of participants, the time factor and context that impacted on the specific findings so it could not be generalised. Further research might explore the lived zoom experiences of administrators, students, and a larger group of faculty over a longer period. 


\section{REFERENCES}

Abbass, A., Arthey, S., Elliott, J., Fedak, T., Nowoweiski, D., Markovski, J., \& Nowoweiski, S. (2011). Webconference supervision for advanced psychotherapy training: A practical guide. Psychotherapy, 48(2), 109-118. doi:10.1037/a0022427 PMID:21639654

Amponsah, S., Badu-Nyarko, S. K., Obodai, G. A. N. S., \& Anane, P. (2019). Learning environment for supporting undergraduate online distance education students. In M. M. Van Wyk (Ed.), Student support toward self-directed learning in open and distributed environments (pp. 78-102). IGI Global.

Buchanan, T., Sainter, P., \& Saunders, G. (2013). Factors affecting faculty use of learning technologies: Implications for models of technology adoption. Journal of Computing in Higher Education, 25(1), 1-11. doi:10.1007/s12528-013-9066-6

Chatwin, J., \& McEvoy, P. (2019). Conversation Analysis (CA) as a tool for exploring interaction in an online video-conferencing based support service. Journal of Enabling Technologies, 13(3), 150-157. doi:10.1108/ JET-11-2018-0051

Cheng, H. J., \& Zhan, H. (2012). Examining pre-service teachers' instructional strategies for technological pedagogical content knowledge via video-conferencing. Journal of Educational Technology Development and Exchange, 5(2), 6. doi:10.18785/jetde.0502.05

Coetzee, N., Maree, D. J., \& Smit, B. N. (2019). The relationship between chronic fatigue syndrome, burnout, job satisfaction, social support and age among academics at a tertiary institution. http://hdl.handle.net/2263/76060

Commonwealth of Learning. (2020). Guidelines on distance education during COVID-19. Burnaby, British Columbia, Canada: Commonwealth of Learning.

Corbera, E., Anguelovski, I., Honey-Rosés, J., \& Ruiz-Mallén, I. (2020). Academia in the time of COVID-19: Our chance to develop an ethics care. Laboratory for the Analysis of social-ecological systems in a globalised world. www.laseg.cat/en/news/22/academia-in-the-time-of-covid-19-our-chance-to-develop-an-ethics-of-care

Cross, T., \& Pollk, L. (2018). Burn Bright, Not Out: Tips for Managing Online Teaching. Journal of Educators Online, 15(3). https://eric.ed.gov/?id=EJ1199109

Demerouti, E., Bakker, A. B., Nachreiner, F., \& Schaufeli, W. B. (2001). The job demands-resources model of burnout. The Journal of Applied Psychology, 86(3), 499-512. doi:10.1037/0021-9010.86.3.499 PMID:11419809

Derryberry, D., \& Tucker, D. M. (1994). Motivating the focus of attention. In P. Niedenthal \& S. Kitayama (Eds.), The heart's eye: Emotional influence in perception and attention (pp. 167-192). New York: Academic. doi:10.1016/B978-0-12-410560-7.50014-4

Halupa, C., \& Bolliger, D. U. (2020). Technology Fatigue of Faculty in Higher Education. Technology, 11(18). Advance online publication. doi:10.7176/JEP/11-18-02

Hines, A., \& Sun, P. (2020). Zoom fatigue: how to make video calls less tiring. https://theconversation.com/ zoom-fatigue-how-to-make-video-calls-less-tiring-137861

Hobbs, R., \& Coiro, J. (2016). Everyone learns from everyone: Collaborative and interdisciplinary professional development in digital literacy. Journal of Adolescent \& Adult Literacy, 59(6), 623-629. doi:10.1002/jaal.502

Hobbs, R., \& Coiro, J. (2019). Design features of a professional development program in digital literacy. Journal of Adolescent \& Adult Literacy, 62(4), 401-409. doi:10.1002/jaal.907

Hockey, G. R. J. (1993). Cognitive-energetical control mechanisms in the management of work demands and psychological health. In A. Baddely \& L. Weiskrantz (Eds.), Attention: Selection, awareness, and control (pp. 328-345). Clarendon Press.

Hofflander, M., Nilsson, L., Eriksén, S., \& Borg, C. (2016). Framing the implementation process of video conferencing in discharge planning-According to staff experience. Informatics for Health \& Social Care, 41(2), 192-209. doi:10.3109/17538157.2015.1008484 PMID:25710202

International Baccalaureate Organization. (2020). Online learning, teaching and education continuity planning for schools. https://www.ibo.org/globalassets/news-assets/coronavirus/online-learning-continuity-planning-en.pdf 
Ip, W. H. (2012). Video conferencing: Advantages and limitations in teaching intercultural communication in foreign language education. Proceedings of the 5th edition of the international conference "ICT for Language Learning.

Jayaraman, R., \& Jothiswaran, V. V. (2020). Web-based platforms for virtual learning. Biotica Research Today, 2(5), 184-186.

Jiang, M. (2020). The reason zoom calls drain your energy. https://www.bbc.com/worklife/article/20200421why-zoom-video-chats-are-so-exhausting

Kubey, R. W., Lavin, M. J., \& Barrows, J. R. (2001). Internet use and collegiate academic performance decrements: Early findings. Journal of Communication, 51(2), 366-382. doi:10.1111/j.1460-2466.2001.tb02885.x

Langford, M., \& Damsa, C. (2020). Online teaching in the time of COVID-19: Academic teachers' experience in Norway. Centre for Experiential Legal Learning (CELL), University of Oslo.

Lawson, S. (2020). Are schools forcing students to install spyware that invades their privacy as a result of the Coronavirus lockdown? https://www.forbes.com/sites/seanlawson/2020/04/24/are-schools-forcing-students-toinstall-spyware-that-invades-their-privacy-as-a-result-of-the-coronavirus-lockdown/\#7b25c085638d

Lazarus, R. S., \& Folkman, S. (1984). Stress, appraisal, and coping. Springer Publishing Company.

Ma'mari, A. L. (2020). Fatigue, burnout, work environment, workload and perceived patient safety culture among critical care nurses. British Journal of Nursing (Mark Allen Publishing), 29(1), 28-34. doi:10.12968/ bjon.2020.29.1.28 PMID:31917951

Macgilchrist, F. (2020). Three stories about edtech after the corona pandemic. TECHLASH, 1, 11-17. https:// der.monash.edu.au/lnm/wp-content/uploads/2020/06/TECHLASH-01-COVID-education.pdf

Marrriam-Webster. (n.d.). Academic. In Merriam-Webster.com dictionary. https://www.merriam-webster.com/ dictionary/academic

Oeppen, R. S., Shaw, G., \& Brennan, P. A. (2020). Human factors recognition at virtual meetings and video conferencing: How to get the best performance from yourself and others. British Journal of Oral \& Maxillofacial Surgery, 58(6), 643-646. Advance online publication. doi:10.1016/j.bjoms.2020.04.046 PMID:32417017

OFCOM. (2014). The Communication Market Report 2014. https://www.ofcom.org.uk/_data/assets/pdf_ file/0031/19498/2014_uk_cmr.pdf

Pettersson, F. (2018). On the issues of digital competence in educational contexts - a review of the literature. Educational Informational Technology, 23(3), 1005-1021. doi:10.1007/s10639-017-9649-3

Reisinger, T. (2020). Zoom security: I've researched problems with video conferencing for years - here's what you need to know. https://theconversation.com/zoom-security-ive-researched-problems-with-video-conferencingfor-years-heres-what-you-need-to-know-136330

Richter, P., \& Hacker, W. (1998). Belastung und Beanspruchung: Stress, Ermüdung und Burnout im Arbeitsleben [Workload and strain: Stress, fatigue and burnout in working life]. Asanger.

Sato, M., Smeaton, K., \& Palmer, T. (2019). "What's Up, Doc?”: Selling Digital Literacy to Academics. Proceedings of the I ATUL Conferences. https://docs.lib.purdue.edu/iatul/2019/bp/8

Shuttleworth, S. (2020). Fagged out: Overwork and sleeplessness in Victorian professional life. Interface Focus, 10(3), 1-10. doi:10.1098/rsfs.2019.0088 PMID:32382401

Spicer, A. (2020). Finding endless video calls exhausting? You're not alone. https://theconversation.com/findingendless-video-calls-exhausting-youre-not-alone-137936

Sudhir, M., Mascarenhas, S., Isaac, J., Alfroukh, J., \& Rahuman, S. A. (2020). Adapting to the need of the hour: Communication skills simulation session using an online platform during COVID-19. MedEdPublish, 9(1), 85. doi:10.15694/mep.2020.000085.1

Tanis, C. J. (2020). The seven principles of online learning: Feedback from faculty and alumni on its importance for teaching and learning. Research in Learning Technology, 28(0). Advance online publication. doi:10.25304/ rlt.v28.2319 
Tlou, G. (2020). Covid-19: Private university students in SA unable to study online due to high data costs. https://www.iol.co.za/the-star/news/covid-19-private-university-students-in-sa-unable-to-study-online-due-tohigh-data-costs-47604061

UN COVID-19 Global Education Coalition. (n.d.). https://www.un.org/sustainabledevelopment/education/

University of Ghana. (2020). No: 4: Roll out of online teaching and learning at UG. http://ug.edu.gh/ announcements/no-4-roll-out-online-teaching-and-learning-ug

University of Ghana. (2020). Overview. https://www.ug.edu.gh/about/overview

Van der Linden, D., \& Eling, P. (2006). Mental fatigue disturbs local processing more than global processing. Psychological Research, 70(5), 395-402. doi:10.1007/s00426-005-0228-7 PMID:15968553

van Wyk, M. M., \& Taole, M. (2015). Research design. In Educational research: An African approach. Oxford University Press.

Vetter, A. (2018). The matrix of convivial technology-assessing technologies for degrowth. Journal of Cleaner Production, 197, 1778-1786. doi:10.1016/j.jclepro.2017.02.195

World Economic Forum. (2020). These are the video apps we're downloading during coronavirus. https://www. weforum.org/agenda/2020/03/infographic-apps-pandemic-technology-data-coronavirus-covid19-tech/

Samuel Amponsah is a Senior Lecturer with a demonstrated history of working at all levels of education. He is the Acting Head of University of Ghana's Distance Education Department. He holds a Doctor of Education degree from the University of South Africa. Before joining the University of Ghana, Amponsah lectured at Esayidi TVET in South Africa and headed the Farming Management Department. Samuel's areas of research interest are distance and online learning and adult learning. He is a fellow of the Global Challenges Research Fund and currently a Co-l for two international projects supported by the Global Challenges Research Fund and the British Council.

Chair of Department, an established researcher in teacher education, published research articles, reading papers at educational conferences, written academic books and chapters in research books, supervised doctoral and masters' degree students, delivered several keynote addresses at teacher education conferences. Awarded the Chancellors' 2013 Award for Excellence in Research. Furthermore, awarded the Scholarship of Teaching and Learning, College of Education (2018). Founder and Editor-in-chief for African Journal of Pedagogy and Curriculum. Michael, K. Kolugu presently teaches Computer Science and IT courses at the Department of Computer Science and Department of Distance Education of the University of Ghana. Prior to his current position, he was a Teaching Assistant and Graduate Assistant at the University of Ghana. He later joined Blue Crest University College as a full-time lecturer and headed the IT Department while also tutoring IT courses on an Off-campus Master Programme from the University of Sunderland. His research interests areas are Computer Vision, Algorithm Optimization, and Machine Learning and its application in varied fields including education.Michael, K. Kolugu presently teaches Computer Science and IT courses at the Department of Computer Science and Department of Distance Education of the University of Ghana. Prior to his current position, he was a Teaching Assistant and Graduate Assistant at the University of Ghana. He later joined Blue Crest University College as a full-time lecturer and headed the IT Department while also tutoring IT courses on an Off-campus Master Programme from the University of Sunderland. His research interests areas are Computer Vision, Algorithm Optimization, and Machine Learning and its application in varied fields including education. 